\title{
Lower periorbital area treatment with Restylane Vital Skinbooster
}

\author{
Denis Souto Valente ${ }^{\mathbf{l}^{*}}$, Rafaela Koehler Zanella ${ }^{2}$, Ângelo Syrillo Preto Neto ${ }^{3}$, \\ Sibelie Souto Valente ${ }^{4}$, Felipe Laranjeira ${ }^{1}$ \\ ${ }^{1}$ Graduate Program in Medicine and Health Sciences, Pontifical University Catholic Rio Grande do Sul School of Medicine (FAMED), Porto Alegre, \\ RS, Brazil \\ ${ }^{2}$ Dermatologic Surgery Department, Mãe de Deus Health System, Porto Alegre, RS, Brazil \\ ${ }^{3}$ Plastic Surgery Department, Mãe de Deus Health System, Porto Alegre, RS, Brazil \\ ${ }^{4}$ Postgraduate Division, Anhanguera Pelotas College, Pelotas, RS, Brazil
}

\begin{abstract}
Facial aging along the periocular area has led to the development of new non-surgical therapies. The lower periorbital area (LPOA) is a vital region for facial rejuvenation and several procedures have been studied to treat it, especially procedures with dermal fillers for volume rebuilding. This manuscript aims to describe a filling technique with hyaluronic acid (HA) along the superficial layer of the lower periorbital area, presenting its effectiveness and safety. Our assessment was made by autonomous observers with pictures of before and after treatment, rated from 0 (no enhancement) to 100 (maximum enhancement). Patients' self-assessment was also performed using a visual analog scale (VAS) based on a 100-mm ruler affording a 0-100 rating. Thirty patients were enrolled in this study. The autonomous observers' evaluation presented a 78.4 mean improvement rate. Patients' self-assessments after the treatment were $0 \%$ reporting no improvement, $6.7 \%$ mild improvement, $23.3 \%$ moderate improvement, $66.7 \%$ great improvement, and 3.3\% maximum improvement. Restylane Vital Skinbooster infiltration at the lower periorbital area proved to be a safe treatment. The described technique is also simple to execute, has a low rate of complications, and produces a high satisfaction rate.
\end{abstract}

Keywords: Soft tissue augmentation; blepharoplasty; dermal fillers; hyaluronic acid

Citation: Valente DS, Zanella RK, Neto ÂSP, Valente SS, Laranjeira F. Lower periorbital area treatment with Restylane Vital Skinbooster. J Surg Dermatol 2016; 1(3): 168-174; http://dx.doi.org/10.18282/jsd. v1.i3.4.

*Correspondence to: Denis Valente, Graduate Program in Medicine and Health Sciences, PUCRS School of Medicine (FAMED), Av Ipiranga 6681, Porto Alegre, RS, 90619-900, Brazil, denisvalentedr@ gmail.com.

Received: $9^{\text {th }}$ November 2015; Accepted: $15^{\text {th }}$ January 2016; Published Online: $19^{\text {th }}$ September 2016

\section{Introduction}

Facial aging along the periocular area has led to the development of new, non-surgical therapies. The lower periorbital area (LPOA) is a vital region for facial rejuvenation and several procedures have been studied to treat it, especially procedures with dermal fillers for volume rebuilding ${ }^{[1-3]}$. Hyaluronic acid (HA) seems to be the most widely used and safest injectable dermal filler. Most of its complications disappear when the product degrades or is destroyed with hyaluronidase. HA also 
rarely induces significant side effects. The LPOA is a challenging area for facial rejuvenation treatments. In order to enhance the silhouette of this region, many authors have proposed treatments using injectable $\mathrm{HA}^{[4,5]}$. The main objective of this manuscript is to describe the technique for LPOA filling using HA at the superficial layer, and to examine its effectiveness and safety.

\section{Materials and methods}

This longitudinal and prospective study was performed at a private clinic from October 2013 to July 2015. Patients were photographed before, and two months after, the procedure. Two independent physicians - a plastic surgeon and a dermatologist - performed assessments by comparing digital pictures prior to and 60 days post-procedure, providing a rating of 'no improvement' $(0)$ to 'maximum improvement' (100). After 60 days during a revision appointment, the patients measured their procedural outcomes by using a visual analog scale (VAS) consisting of a 100-mm ruler. The score was set by measuring the distance in $\mathrm{mm}$ along the $100-\mathrm{mm}$ line between 'no improvement' to 'maximum improvement' marks, starting from 0 to the patient's marking, with ratings ranging from $0-100$. Lower ratings indicate reduced enhancement. In assessing the VAS distribution, the following intervals were used to state the outcomes: no improvement $(0-10 \mathrm{~mm})$, mild improvement $(11-40 \mathrm{~mm})$, moderate improvement $(41-70 \mathrm{~mm})$, great improvement (71-89 mm), and maximum improvement (90-100 mm). This outcome scale has previously been successfully described $^{[6]}$. Following the data collection, the outcomes were analyzed using descriptive statistics via the statistical software SPSS 18 (IBM, USA).

\section{Procedural techniques}

Pliaglis (G Production, Canada) was used as topical anesthesia $20 \mathrm{~min}$ before treatment. Asepsis with chlorhexidine gluconate was performed and the patient was positioned at a $45^{\circ}$ inclination from the longitudinal axis looking towards their own forehead, in an attempt to make their palpebromalar fat compartment visible. HA elected for this study was Restylane Vital (QMed AB, Sweden). HA was injected above the periorbital muscle on both sides, starting at the infraorbital edge in the tea groove area going up to the cranial edge of the lower eyelid, via a fan pattern with slow and steady movements using a 30-G needle. Retroinjection was used to allocate the HA in the subdermal layer by means of a fan pattern from the sagittal medio-pupillar point at the lower orbital rim. Figure 1 demonstrates the HA allocation technique. HA is distributed gently and molded using finger pressure against the hard surface of the orbital rim for correct product distribution in order to avoid lump formation or overcorrection.

In order to prevent swelling and bruising, cold packs were applied after the treatment, and physical exercise, exposure to high temperatures, and local massage were forbidden for $48 \mathrm{~h}$. All of the patients were photographed under common conditions before and 60 days after the procedure. Before and after pictures for some of the patients are presented in Figures 2-4. Additional facial aesthetic therapies such as the botulinum toxin, laser, or chemical peels were not permitted for these patients in the following 60 days. Patients were evaluated at 15 and 60 days after the treatment in order to analyze the side effects.

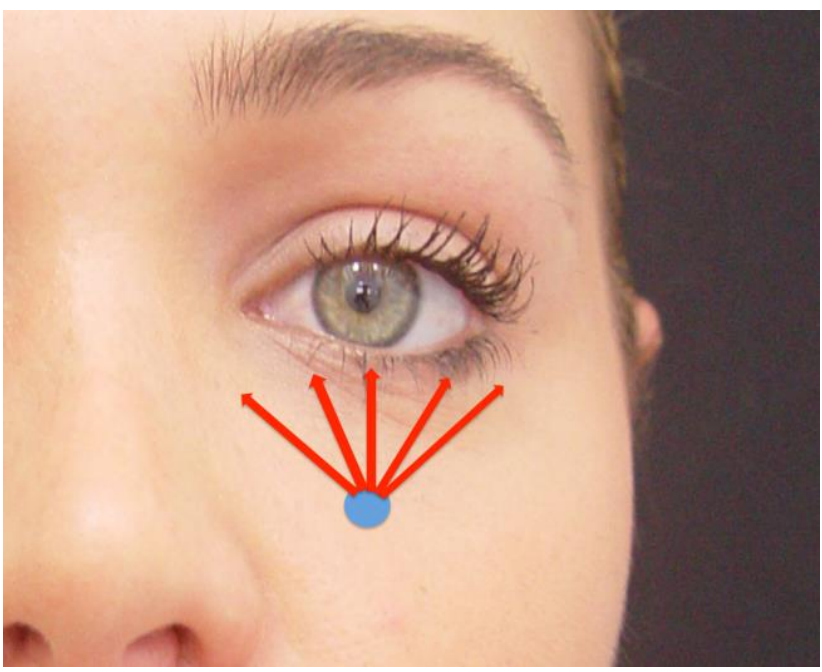

Figure 1. Schematic distribution of $\mathrm{HA}^{[6]}$

\section{Ethics statement}

Patients were made aware of the study protocol and signed a written informed consent before the study and patient enrollment began. This study was designed following the 2000 Edinburgh revision of the Declaration of Helsinki and the applicable ICH guidelines, as well as the Guidelines on Research Practice. This study did not alter the medical assistance provided to the patients. All of the participants provided written informed consent before the treatment, allowing use of data as well as before and after images for this manuscript. 


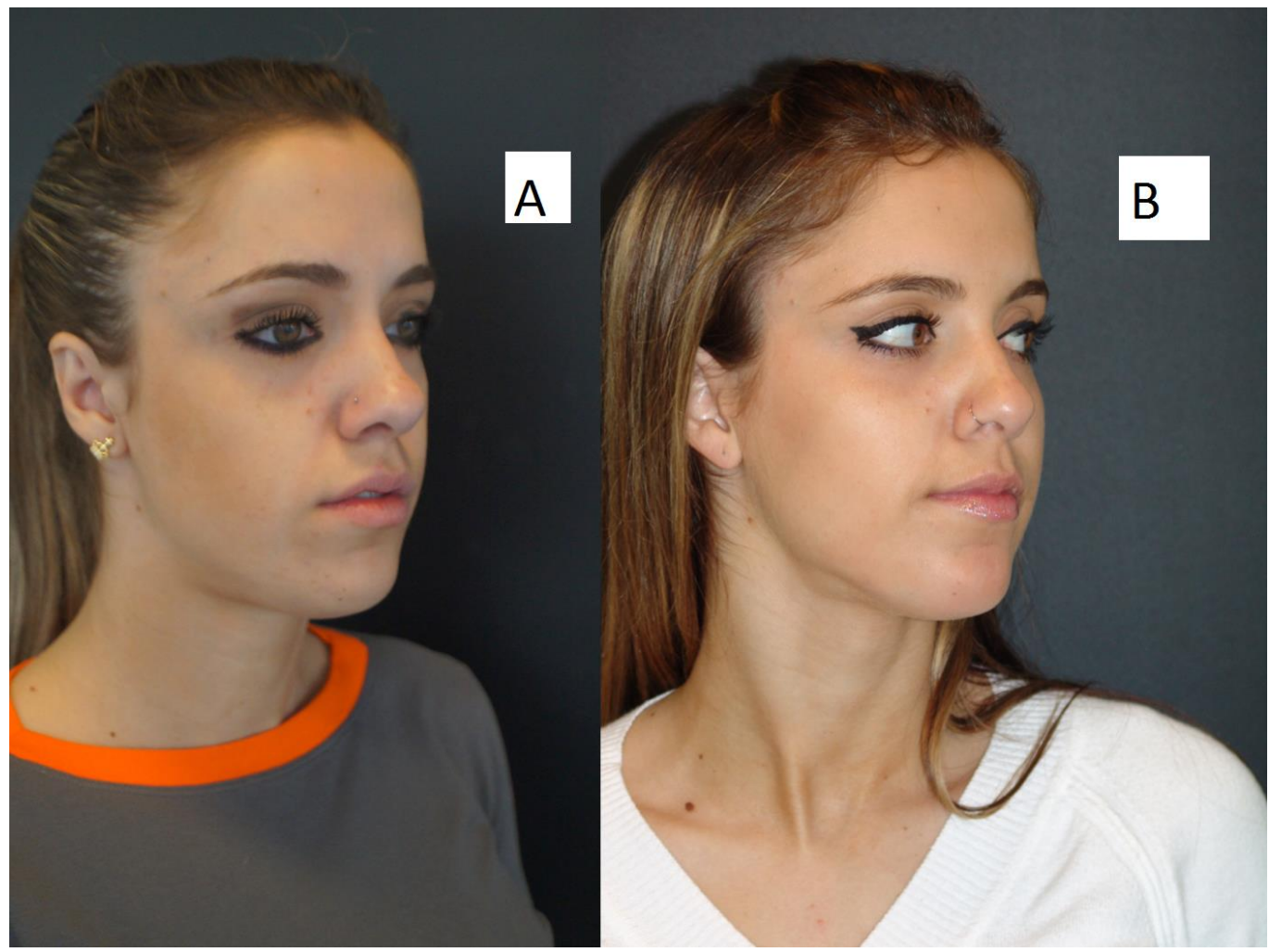

Figure 2. 33-year-old female patient (a) before the procedure; (b) 2 months after the procedure

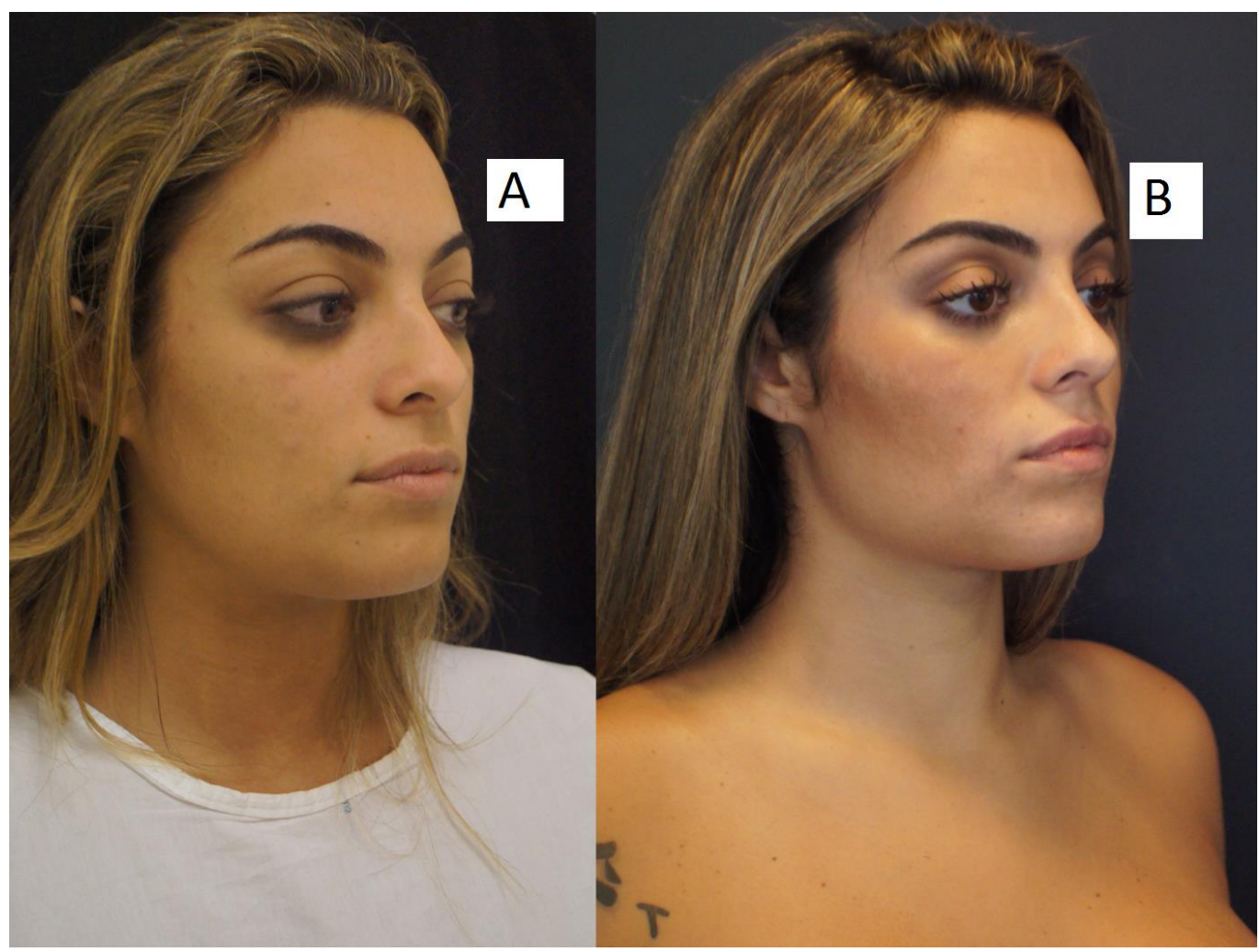

Figure 3. 25-year-old female patient (a) before the procedure; (b) 2 months after the procedure 


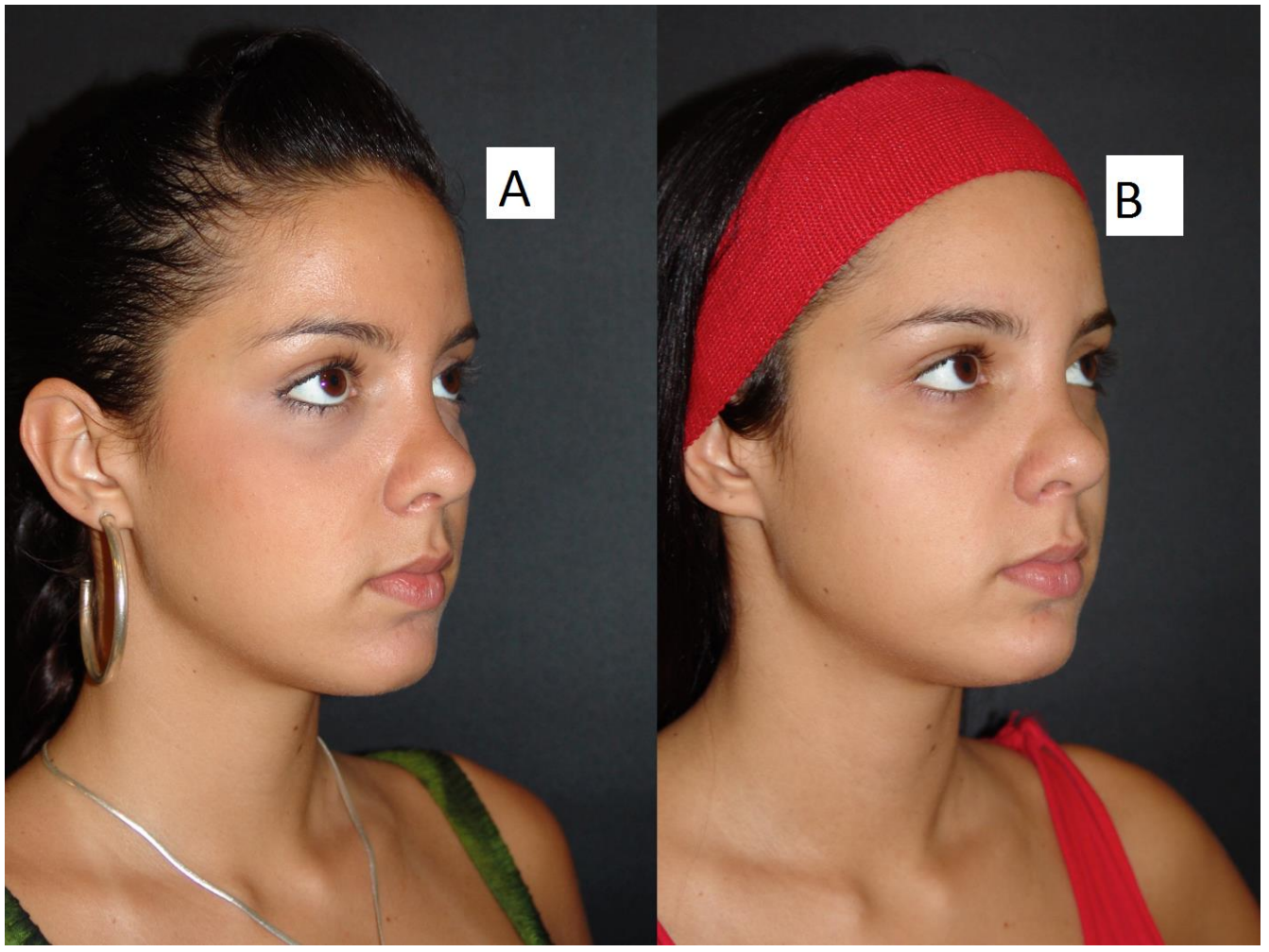

Figure 4. 29-year-old female patient (a) before the procedure; (b) 2 months after the procedure

\section{Results}

A total of 30 patients participated in this research. The average age was 37.5 years old, and $93.3 \%$ of them (28 patients) were female. Table 1 shows their individual characteristics. We present the results observed following a single HA application. The mean injected amount of HA per side was $0.42 \mathrm{~mL}$, varying from $0.3-0.5 \mathrm{~mL}$. The outcomes stated by the independent physicians presented a 78.4 mean improvement score. Patient's evaluations after the treatment were $0 \%$ for no improvement, $6.7 \%$ mild improvement, $23.3 \%$ moderate improvement, $66.7 \%$ great improvement, and $3.3 \%$ maximum improvement. Three patients presented mild and transient ecchymosis. One patient experienced persistent swelling at the LPOA in the following days, with spontaneous resolution after three weeks. One patient presented an overcorrection, requiring local treatment with hyaluronidase 1000 RTU. All individuals were able to return to work a day after the procedure. None of the subjects presented lumps, nodules, pain, skin color changes, or Tyndall effect in the follow-up visits.
Table 1. Patient characteristics

\begin{tabular}{lc}
\hline & Mean \\
\hline Age (years) & 37.5 \\
Male (\%) & 6.7 \\
Caucasian (\%) & 100 \\
BMI $\left(\mathrm{kg} / \mathrm{m}^{2}\right)$ & 22.6 \\
Smoking $(\%)$ & 12.1 \\
Post-procedure evaluation (days) & 61.8 \\
\hline
\end{tabular}

\section{Discussion}

Many therapies for LPOA treatment have been published. Some manuscripts have described the use of dermal fillers and discussed the appropriate dermal filler for very tenuous and vascularized skin on this anatomical area. HA is highlighted as the most advantageous - due to its homogeneous texture, low allergenic potential, and simplicity in application - by providing lower complication rates and predictable results when applied to the skin 
layer. Restylane Vital Skinbooster is a stabilized nonanimal-based HA designed for skin hydration and rejuvenation. It was developed to meliorate skin structure, restore its hydrobalance, and improve elasticity ${ }^{[7-9]}$.

LPOA has an extraordinarily slim dermis, composed of loose connective tissues virtually absent in the pretarsal, lateral, and medial eyelids ligaments, adhering to the fibrous underlying structure. Due to the unique anatomical features of LPOA, we did not find any consensus in literature regarding the most suitable technique for HA application along this area. Major precautionary measures should be taken to avoid an injection through the orbital septum. This injury could cause an intraorbital fat herniation or an inadvertent injection at the extraocular muscles. In cases of immediate hematoma, the procedure must be suspended to minimize the risk of vascular embolism. HA injection must be relatively quick, to avoid injection edema which can distort local anatomy ${ }^{[10-12]}$. The technique described here allows a constant visualization of the needle in order to assert that the HA is being injected along the correct layer.

Landau and Fagien stated, "The first product designed specifically for skin-boosting purposes was Restylane Vital. The product comprises small particles stabilized smooth and relatively thin NASHA gel $(20 \mathrm{mg} / \mathrm{mL}) \ldots$ The investigators found that micropuncture injections of Restylane Vital significantly increased skin elasticity and created positive impact on skin surface roughness. Since great improvement in these parameters was evident 24 weeks after the last injection session, the authors concluded that placement of HA into the dermis enhanced biosynthesis of new dermal compounds and is not only due to better skin hydration, as initially expected.. ${ }^{,[13]}$ They also declared that, "Recent clinical observations demonstrated the persistence of the filling effect longer than the biological availability of the filler. Stimulation of new collagen by cross-linked HA and up-regulation of elastin has been suggested as possible explanation to this observation and has been supported experimentally. Cross-linked HA substitutes for fragmented collagen in restoring extracellular matrix are required for normal activity of fibroblasts, such as collagen and elastin production....Boosting of facial skin through fibroblast activation is a new indication for HA-based products. Injectable HA has also been recently registered in Europe as agents specific for the improvement of skin quality (Restylane Skinboosters). ${ }^{,[13]}$ Additionally, Landau and Fagien also reported, "Improvement of skin quality by injectable cross-linked HA has evolved in the last years, as an additional indication of HA-based dermal fillers use. The procedure hydrates the dermis and creates stable extracellular matrix to support intradermal fibroblasts structure that is essential for their normal function....[F]urther research will identify the relative role of each component, so that we can establish best practices, products selection, and techniques for optimal results.. ${ }^{[13]}$ For those reasons, Restylane Vital Skinbooster was elected for this research.

In addition to being easy to store, non-immunogenic and biocompatible, and especially for its solubility with hyaluronidase, HA presents a lot of advantages over other dermal fillers. This specific characteristic permits the removal of the product in case of chronic adverse reaction or the correction of excessive volume injections. Only one subject in this research required hyaluronidase following the application in order to correct irregularities. Other often-described complications such as migraine, lymphedema unresponsive to hyaluronidase, local cellulitis, and persistent erythema were not found in our research. Another claim of complication, fortunately rare, is blindness following dermal filler injections. There are a few published safety measures to prevent ophthalmic artery embolization, such as applying small amounts of filler, avoiding filler application near the inner corner of the eye in order to prevent angular arteries, using microcannulas with blunt tips, and precluding bolus injection technique ${ }^{[14-17]}$.

Many studies used subjective methods of evaluation to assess aesthetic outcomes. VAS is an objective method that must be marked by the study subjects; otherwise, it would only be a verbal rating system instead of a visual scale. It estimates the problematic dimensions of patients' expectations and the obtained results. We used it in our research in order to transform patient's self-perception after the procedure into a numeric expression for statistical analysis. Using these tactics, we can accord a validated mechanism for the aesthetic procedure assessment ${ }^{[18]}$.

In subjects with significantly sagging skin, our technique is contraindicated; blepharoplasty must be performed for tissue removal and correction of the orbital septum. HA can be used as an ancillary procedure, but is suitable only for correcting the loss of regional volume in these patients.

Our study's interpretation is restrained due to the lack of a control group. Our research is based on a self-selected group seeking treatment for LPOA in a private clinic. It would be very arduous to recruit volunteers for a randomized placebo-controlled trial. Wang et al. studied photodamaged forearm skin injected with HA (Restylane) or saline solution (Restylane vehicle). HA, and not the 
saline injections, was related with type I collagen deposition around the injection site ${ }^{[19]}$. Although both studies are not strictly equivalent, there is no reason to believe that LPOA skin would respond contrarily to forearm skin. Assuming this theory, Wang's study provided strong evidence that HA can cause positive dermal alterations, hence ultimately generating better skin.

Theoretically, HA is absorbed one year after its application. However, different studies have shown the presence of residual volume remaining for more than 12 months. Attraction of water molecules into the extracellular matrix is probably involved in the mechanism by which HA promotes this residual increased volume effect, thus improving dermis elasticity by neocollagenesis stimulation. Our research was not aimed to assess the duration of the gained effect. Hereafter, we expect to use the same patient database to assert the outcome's duration.

\section{Conclusion}

Our data suggests that the use of Restylane Vital Skinbooster along the LPOA presents a low risk of complications, is easy to perform, and provides a high satisfaction degree. This procedure can be safely performed in young patients with no sagging skin and as an ancillary procedure to eyelid surgery.

\section{Conflict of interest}

The senior author acts at the Speaker's Bureau membership of Galderma Incorporation, distributor of Restylane products. None of the authors have ownership interest (stocks, stock options, or other ownership interest), are full-time/part-time employees or are Honorarium recipients of any company related with the products presented in the research.

\section{References}

1. Carruthers J, Carruthers A. Social significance of the eyebrows and periorbital complex. J Drugs Dermatol 2014; 13(1 Suppl): s7-s11.

2. Kane MA. Nonsurgical periorbital and brow rejuvenation. Plast Reconstr Surg 2015; 135(1): 63-71. doi: 10.1097/ PRS.0000000000000831.

3. Pilkington SJ, Belden S, Miller RA. The tricky tear trough: A review of topical cosmeceuticals for periorbital skin rejuvenation. J Clin Aesthet Dermatol 2015; 8(9): $39-47$.

4. Stutman RL, Codner MA. Tear trough deformity: Review of anatomy and treatment options. Aesthet Surg J 2012; 32(4): 426-440. doi: 10.1177/1090820X12442372.

5. Huber-Vorländer J, Kürten M. Correction of tear trough deformity with a cohesive polydensified matrix hyaluronic acid: A case series. Clin Cosmet Investig Dermatol 2015; 8: 307-312. doi: 10.2147/CCID.S84117.

6. Valente DS, Zanella RK, Valente SS. Hyaluronic acid associated with abobotulinumtoxin use along the lower periorbital area: A prospective study. J Clin Exp Dermatol Res 2016; 7(1): 323. doi: 10.4172/2155-9554.1000323.

7. Cohen JL, Dayan SH, Brandt FS, Nelson DB, Axford-Gatley RA, et al. Systematic review of clinical trials of small- and large-gel-particle hyaluronic acid injectable fillers for aesthetic soft tissue augmentation. Dermatol Surg 2013; 39(2): 205-231. doi: 10.1111/dsu.12036.

8. Raspaldo H, Gassia V, Niforos FR, Michaud T. Global, 3-dimensional approach to natural rejuvenation: Part 1 Recommendations for volume restoration and the periocular area. J Cosmet Dermatol 2012; 11(4): 279-289. doi: 10.1111/jocd.12003.

9. Lee S, Yen MT. Injectable hyaluronic acid fillers for periorbital rejuvenation. Int Ophthalmol Clin 2013; 53(3): 1-9. doi: 10.1097/IIO.0b013e31828c0499.

10. Sharad J. Dermal fillers for the treatment of tear trough deformity: A review of anatomy, treatment techniques, and their outcomes. J Cutan Aesthet Surg 2012; 5(4): 229-238. doi: 10.4103/0974-2077.104910.

11. Berros P, Lax L, Bétis F. Hyalurostructure treatment: Superior clinical outcome through a new protocol - A 4-year comparative study of two methods for tear trough treatment. Plast Reconstr Surg 2013; 132(6): 924e-931e. doi: 10.1097/PRS.0b013e3182a80683.

12. Bravo BSF, Rocha CRMD, Bastos JTD, Silva PMC. Comprehensive treatment of periorbital region with hyaluronic acid. J Clin Aesthet Dermatol 2015; 8(6): 30-35.

13. Landau M, Fagien S. Science of hyaluronic acid beyond filling: Fibroblasts and their response to the extracellular matrix. Plast Reconstr Surg 2015; 136(5 Suppl): 188S195S. doi: 10.1097/PRS.0000000000001823.

14. Valente DS, Zanella RK. Fillers complications for HIV/AIDS lipoatrophy treatment. Rev Bras Cir Plást 2015; 30(2): 335-336. doi: 10.5935/2177-1235.2015RBCP0160.

15. Nácul AM, Valente DS. Complications after polymethylmethacrylate injections. Plast Reconstr Surg 2009; 124(1): 342-343. doi: 10.1097/PRS.0b013e3181a83ac2.

16. Beleznay K, Carruthers JD, Humphrey S, Jones D. Avoiding and treating blindness from fillers: A review of the world literature. Dermatol Surg 2015; 41(10): 1097-1117. doi: 10.1097/DSS.0000000000000486. 
17. Woodward J, Khan T, Martin J. Facial filler complications. Facial Plast Surg Clin North Am 2015; 23(4): 447-458. doi: 10.1016/j.fsc.2015.07.006.

18. Ferreira MC. Evaluation of results in aesthetic plastic surgery: Preliminary observations on mammaplasty. Plast Reconstr Surg 2000; 106(7): 1630-1635. doi: 10.1097/
00006534-200012000-00032.

19. Wang F, Garza LA, Kang S, Varani J, Orringer JS, et al. In vivo stimulation of de novo collagen production caused by cross-linked hyaluronic acid dermal filler injections in photo-damaged human skin. Arch Dermatol 2007; 143(2): 155-163. doi: 10.1001/archderm.143.2.155. 